\title{
Recessive mitochondrial ataxia syndrome
}

INSERM

\section{Source}

INSERM. (1999). Orphanet: an online rare disease and orphan drug data base. Recessive mitochondrial ataxia syndrome. ORPHA:94125

Recessive mitochondrial ataxia syndrome is a rare, mitochondrial DNA maintenance syndrome characterized by early-onset cerebellar ataxia, and variable combination of epilepsy, headache, dysarthria, ophthalmoplegia, peripheral neuropathy, intellectual disability, psychiatric symptoms and movement disorders. 